\title{
Galileo NIMS Observations of Io
}

\author{
Ashley Gerard Davies \\ Jet Propulsion Laboratory-California Institute of Technology, 4800 Oak \\ Grove Drive, Pasadena, CA 91109 \\ Sylvain Douté \\ IGPP, University of California, Los Angeles, 405 Hilgard, Box 951567, \\ Los Angeles, CA 90095
}

\begin{abstract}
The Near Infrared Mapping Spectometer (NIMS) on the Galileo spacecraft has been observing the volcanic Jovian satellite Io at regular intervals since June 1996. These infrared observations have allowed detailed mapping of the distribution and grain size of sulfur dioxide on Io's surface, identification of volcanic centers, mapping of the distribution of hot spots, and investigations into the style and evolution of individual volcanic cruptions.
\end{abstract}

\section{Introduction}

The Galileo spacecraft entered orbit around Jupiter in December 1995, and since June 1996 has been regularly observing Io, the most volcanically active body in the Solar System. On board the spacecraft is a suite of instruments capable of observing Io over a wide wavelength range. The Near Infrared Mapping Spectrometer (NIMS) is a moving grating spectrometer, sensitive at 408 wavelengths from 0.7 to $5.2 \mu \mathrm{m}$. NIMS objectives at Io are to study surface composition, the thermal output from volcanoes, and map hot spot variability.

NIMS observations can be grouped into dayside (compositional) and nightside (thermal), and also by image scale. Between June 1996 and September 1999 115 global observations were obtained at low spatial resolutions (typically 100 to $300 \mathrm{~km}$ per pixel) but mostly with high spectral resolution. From the dayside observations $\mathrm{SO}_{2}$ distributions could be measured, on a broad scale. Regional observations were obtained during the close flybys of Io starting in October 1999 (orbit I24) with resolutions of typically $25 \mathrm{~km} / \mathrm{pixel}$, and local observations at high resolution (typically $0.5-2 \mathrm{~km} / \mathrm{pixel}$ ) were obtained of selected targets, including Loki, Prometheus, Chaac, and Pele (Lopes-Gautier et al. 2000). The regional and local observations obtained during the first three close flybys (32 observations in total) are at low spectral resolution (12 wavelengths across the wavelength range) as the grating in the NIMS instrument stuck, prior to the I24 close flyby. Attempts to restore movement have so far proved unsuccessful. This restricts spectroscopy, but still allows band-depth mapping and determination of temperatures. 


\section{Surface composition}

The bright colors that give Io its distinctive appearance suggest an abundance of sulfur-bearing compounds such as $\mathrm{SO}_{2}$, native and short-chain sulfur, and sulfur oxides (Geissler et al. 1999). None of these compounds have been clearly identified, except $\mathrm{SO}_{2}$, due to its numerous and distinctive absorption bands in the NIR (Howell et al. 1984; Carlson et al. 1997), and sulfur, detected in the Pele plume (Spencer et al. 2000). Sulfur is probably a common volcanic gas given that red deposits, probably of red $\mathrm{S}_{2}$ or $\mathrm{S}_{4}$ molecules, are often seen at active volcanic sites (Geissler et al. 1999).

When carrying out spectral analysis of NIMS Io observations, it is difficult to escape the all-pervading influence of sulphur dioxide. Nevertheless, the form of the $\mathrm{SO}_{2}$ deposits are revealing in themselves. Distribution and grain size maps of $\mathrm{SO}_{2}$ frost deposits have been made from analyses of NIMS observations at the global scale, obtained during the G2 to E16 orbits and covering $75 \%$ of Io surface (Douté et al. 2001). These frost deposits can be used as tracers of the various parts of the $\mathrm{SO}_{2}$ cycle. Global mosaics are produced that map $\mathrm{SO}_{2}$ frost coverage and mean grain size. At the scale of the $\mathrm{G} 2-\mathrm{E} 16$ observations $\mathrm{SO}_{2}$ deposits are omnipresent on Io's surface, but mainly concentrated in several large areas, centered at medium latitudes. These $\mathrm{SO}_{2}$-rich regions (with surface coverages higher than $60 \%$ ) show a longitudinal correlation with volcanic plumes located lower in latitude suggesting that these plumes are the principal sources of $\mathrm{SO}_{2}$ gas. This is more clearly seen in high-resolution NIMS images of Io's surface obtained during the close flybys (Lopes-Gautier et al. 2000). After a possible dynamic condensation around the plumes or at the equator, the gas is remobilized by solar or thermal (volcanic) flux and flows, for the most part, latitudinally towards the coldest and nearest regions devoid of hot spots. The correlation of the distribution and grain size mosaics distinguishes four different $\mathrm{SO}_{2}$ physical units that indicate relative regional variations of condensation, metamorphism and sublimation. Finally, a comparison with Voyager ultraviolet (McEwen et al. 1988) and Galileo visible observations (Geissler et al. 1999) reveal some molecular contamination of $\mathrm{SO}_{2}$ at medium and high latitudes, and that these $\mathrm{SO}_{2}$ deposits may be optically thin (Douté et al. 2001).

The aquisition of high-resolution NIMS images have been particularly valuable for mapping $\mathrm{SO}_{2}$. For example, in regional observations the distribution of $\mathrm{SO}_{2}$ in the deposition ring around Prometheus is seen to be larger than the visible white counterpart: this suggests that Prometheus emits several different compounds (such as $\mathrm{S}_{8}$ ) in the gas phase, that condense at a distance from the vent inversely proportional to volatility (Lopes-Gautier et al. 2000). The high-resolution NIMS observations show that the red deposits in the vicinity of volcanic centers (e.g., Culann) were also rich in $\mathrm{SO}_{2}$.

\section{Monitoring volcanic thermal emission}

Silicate volcanic activity appears to be ubiquitous on Io (McEwen et al. 1997, 1998) and NIMS is an excellent instrument for measuring volcanic thermal emission. Various models of volcanic thermal emission have been applied to NIMS data. 
NIMS determined that silicate temperature volcanism was taking place on Io at Zamama, from a two-temperature fit to data obtained during a night-side observation during Galileo's first orbit (Davies et al. 1997). This yielded a liquidus temperature of at least $1100 \mathrm{~K}$, beyond the range of sulfur volcanism. The high temperature and area (less than $0.1 \mathrm{~km}^{2}$ ) well-matched the temperature and area derived fom a SSI eclipse image obtained during the same orbit. Using two-temperature fits, other NIMS hot spots have been found to have areas at silicate liquidus temperatures (Davies, 2000; Davies et al. 2000a). Multipletemperature fits using silicate cooling models (e.g., Davies 1996) yield ages of eruptions and rates of areal coverage. Most of the volcanoes on Io exhibit relatively quiescent eruption styles, indicative of relatively low viscosity magmas. Volumes of eruption are typically tens of $\mathrm{m}^{3}$ per second, with flow thicknesses of order $1 \mathrm{~m}$ (Davies et al. 2000b). With a characteristic slope towards longer wavelengths, the thermal spectra of these eruptions indicate a laminar flow emplacement regime. SSI high-resolution images show that flows at Prometheus and Amirani are akin to terrestrial pahoehoe flow fields (McEwen et al. 2000).

Not all volcanoes behave in this low-key manner. Eruptions in other locations exhibit a more vigorous emplacement mechanism. Galileo observed a large eruption at Pillan in September 1997 (orbit C9). Fortuitously, both NIMS and SSI observed this eruption in darkness during orbit C9. A dual-filter SSI observation yielded a magma temperature in excess of $1700 \mathrm{~K}$ (McEwen et al. 1998). This was the first indication of the presence of high temperature, ultrabasic magmas on Io. Detailed modelling of the combined NIMS and SSI dataset has yielded a better-constrained minimum temperature of $1870 \mathrm{~K}$, a melting temperature comparable to the terrestrial Commondale komatiites (Davies et al. 2000a). This eruption type has not been seen on Earth since the Archean. PostC9 observations revealed new $8-10 \mathrm{~m}$ thick flows covering $5600 \mathrm{~km}^{2}$ (Williams et al. 2000). Modeling of the eruption yielded average mass eruption rates in excess of $10^{3} \mathrm{~m}^{3} \mathrm{~s}^{-1}$, implying peak mass eruption rates an order of magnitude greater (Davies et al. 2000a). Subsequently, NIMS watched as the thermal emission from Pillan decayed as the emplaced flows cooled.

Pele is an equally fascinating volcano, the site of a large plume and red and black plume deposits. Pele consistently emits a preponderance of thermal energy at short NIMS wavelengths over long periods of time. The volume of new, hot material that has to be constantly erupted in order to produce this short IR thermal flux would normally be expected to produce extensive flows, except that with time, there is no evidence of an expected large cooling flow unit. In some way the activity at Pele is strongly confined areally. The most likely explanation is that Pele is an active lava lake, on a scale much larger than those currently active on Earth (Davies et al. 2000a). This analysis was only possible due to good temporal coverage of Io's volcanic activity.

Loki remains enigmatic. Periodic huge eruptions resurface the caldera floor, but the exact nature of the resurfacing mechanism has not yet been identified.

\section{Distribution of hot spots and future observations}

To date, Galileo has observed 97 main volcanic centers on Io: there may be considerably more smaller sites (Blaney et al. 2000; Lopes-Gautier et al. 2000). 
The distribution of active volcanic centers generally shows no obvious correlation with latitude or longitude, with the exception of a lack of volcanoes at very high latitudes. The distribution of active volcanic centers is not well correlated with predictions of surface heat flow patterns derived from the aesthenosphere and deep mantle dissipation models, although the apparent deficit of active centers at high latitudes are consistent with the aesthenosphere model (Lopes-Gautier et al. 1999). High resolution images have been obtained with NIMS in 1999 and 2000. Future observations include, at high resolutions, observations of the Jupiter facing hemisphere and night-side maps of volcanic centers, including Loki and Pele.

\section{Acknowledgements}

The research described in this paper was carried out at the Jet Propulsion Laboratory, California Institute of Technology, under contract with the National Aeronautics and Space Administration.

\section{References}

Blaney, D. B. et al. 2000, Myriads of small hot spots on Io, JGR, submitted

Carlson, R. W. et al. 1997, GRL, 24, 2479

Davies, A. G. 1996, Icarus, 124, 45

Davies, A. G. 2000, Minimum liquidus temperatures from two-temperature fits to Galileo NIMS data, GRL, submitted

Davies, A. G. et al. 1997, GRL, 24, 2447

Davies, A. G. et al. 2000a, Thermal signature, eruption style and eruption evolution at Pele and Pillan on Io, JGR, submitted

Davies, A. G. et al. 2000b, Icarus, 148, 212

Douté, S. et al. 2001, Icarus, 149, 107

Geissler, P. et al. 1999, Icarus, 140, 265

Howell R. et al. 1984, Icarus, 57, 83

Lopes-Gautier, R. et al. 1999, Icarus, 140, 243

Lopes-Gautier, R. et al. 2000, Science, 288, 1201

McEwen, A.S. et al. 1988, Icarus, 75, 450

McEwen, A.S. et al. 1997, GRL, 24, 2443

McEwen, A. S. et al. 1998, Science, 281, 87

McEwen, A.S. et al. 2000, Science, 288, 1193

Spencer, J. et al. 2000, Science, 288, 1208

Williams, D. A. et al. 2000, The July 1997 eruption of Pillan Patera on Io: implications for ultrabasic lava flow emplacement, JGR, submitted 